\title{
Unintended pregnancy by the numbers: A look at Kenya
}

Carol Mukiira

Joyce Mumah

Caroline W. Kabiru

Chimaraoke O. Izugbara

Follow this and additional works at: https://knowledgecommons.popcouncil.org/departments_sbsr-rh

Part of the Health Policy Commons, International Public Health Commons, Maternal and Child Health Commons, Public Health Education and Promotion Commons, and the Women's Health Commons How does access to this work benefit you? Let us know!

\section{Recommended Citation}

Mukiira, Carol, Joyce Mumah, Caroline W. Kabiru, and Chimaraoke O. Izugbara. 2014. "Unintended pregnancy by the numbers: A look at Kenya," fact sheet. Nairobi: African Population and Health Research Center. 


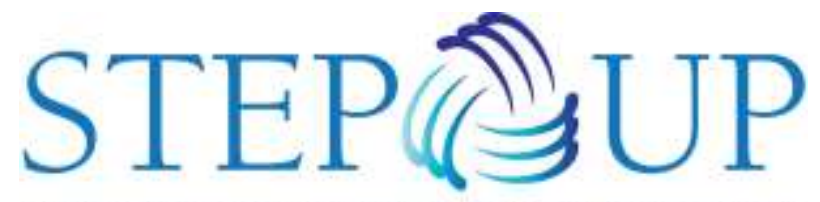

FACT SHEET

\section{UNINTENDED PREGNANCY BY THE NUMBERS}

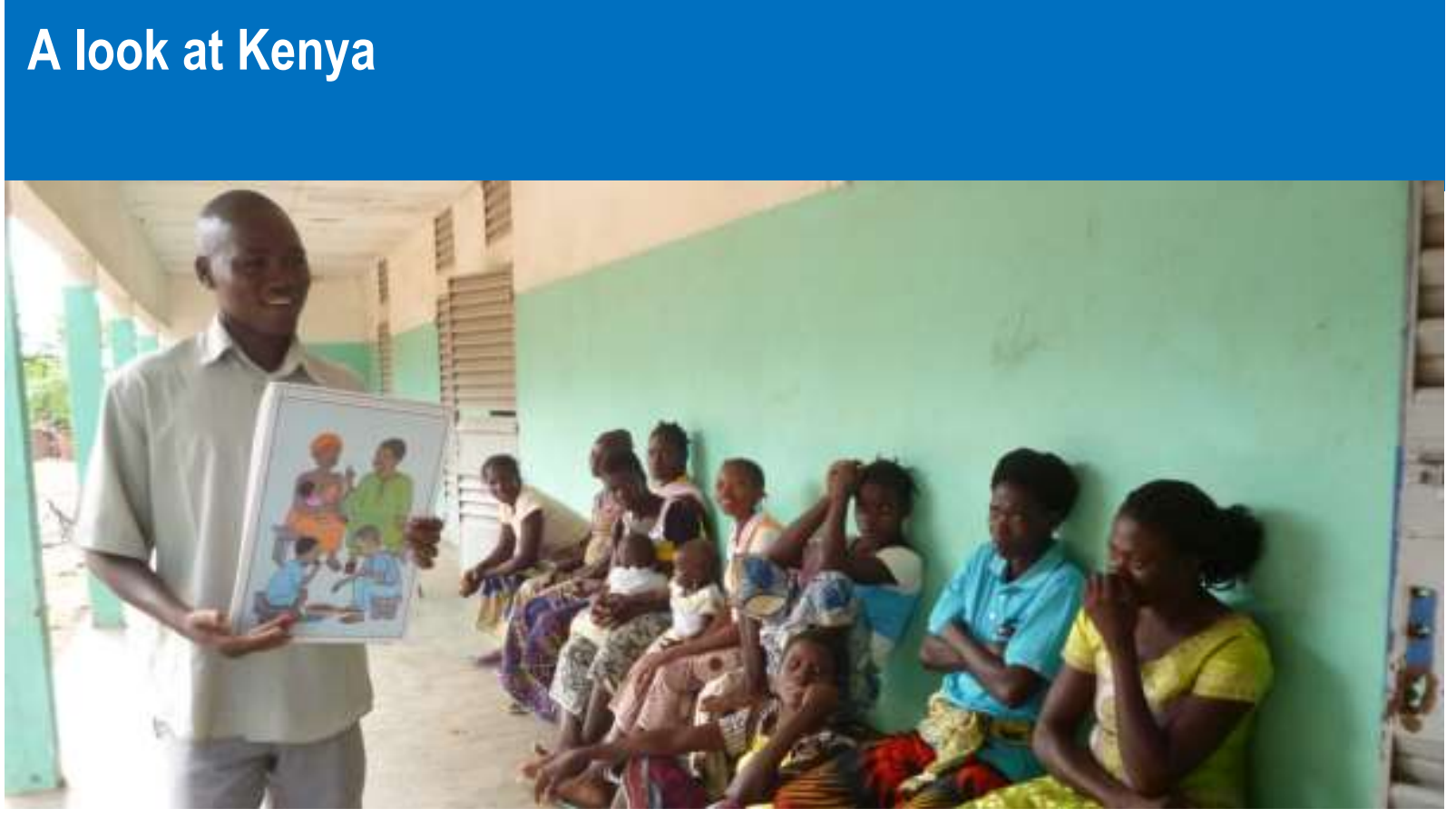

\section{Summary}

This fact sheet summarizes data collated during the development of the STEP UP Country Profile Report on Unintended Pregnancies for Kenya, which presents a range of key evidence aimed at informing readers about the trends, magnitude, determinants and consequences of unintended pregnancy in Kenya. The data shown in this fact sheet are drawn mainly from the 1993, 1998, 2003 and 2008/09 Kenya Demographic and Health Surveys (KDHS) as well as other secondary sources.

Suggested citation: Mukiira C, Mumah, J, Kabiru, CW., and Izugbara, C. 2014. "Unintended Pregnancy by the Numbers: A look at Kenya" STEP UP Fact Sheet. Nairobi: African Population and Health Research Center 
Table 1: Trends in unintended pregnancy $(\%)$

\begin{tabular}{|l|c|c|c|c|}
\hline Type of Unintended Pregnancy & $\mathbf{1 9 9 3}$ & $\mathbf{1 9 9 8}$ & $\mathbf{2 0 0 3}$ & $\mathbf{2 0 0 8 - 0 9}$ \\
\hline Mistimed (wanted later) & 34.2 & 37.2 & 24.9 & 25.5 \\
\hline Unwanted (wanted no more) & 16.7 & 11.1 & 19.6 & 17.1 \\
\hline Unintended pregnancies & 50.9 & 48.3 & 44.5 & 42.6 \\
\hline
\end{tabular}

Source: Kenya DHS, 1993, 1998, 2003, 2008-09

Table 1a: Mistimed pregnancy by selected background characteristics (\%)

\begin{tabular}{|c|c|c|c|c|}
\hline $\begin{array}{l}\text { Background } \\
\text { Characteristics }\end{array}$ & 1993 & 1998 & 2003 & 2008-09 \\
\hline \multicolumn{5}{|l|}{ Age } \\
\hline $15-24$ & 41.3 & 42.7 & 26.9 & 30.9 \\
\hline $25-34$ & 31.1 & 33.9 & 24.5 & 22.2 \\
\hline$>35$ & 14.3 & 22.2 & 11.3 & 12.4 \\
\hline \multicolumn{5}{|l|}{ Education } \\
\hline No education & 24.2 & 27.0 & 12.9 & 17.4 \\
\hline Primary & 36.8 & 40.0 & 28.2 & 28.0 \\
\hline Secondary + & 35.9 & 34.2 & 23.6 & 23.5 \\
\hline \multicolumn{5}{|l|}{ Wealth } \\
\hline Low & 32.7 & 38.4 & 25.6 & 26.8 \\
\hline Medium & 38.0 & 36.6 & 25.0 & 28.3 \\
\hline High & 34.0 & 35.9 & 24.2 & 23.0 \\
\hline \multicolumn{5}{|l|}{ Residence } \\
\hline Urban & 31.3 & 35.5 & 24.7 & 20.4 \\
\hline Rural & 34.6 & 37.6 & 25.0 & 26.7 \\
\hline \multicolumn{5}{|l|}{ Region } \\
\hline Nairobi & 36.0 & 29.3 & 27.8 & 14.5 \\
\hline Central & 30.9 & 29.5 & 18.0 & 19.4 \\
\hline Coast & 30.3 & 39.6 & 22.9 & 6.9 \\
\hline Eastern & 41.8 & 36.9 & 25.1 & 22.6 \\
\hline Nyanza & 27.3 & 34.3 & 32.8 & 18.2 \\
\hline Rift Valley & 34.0 & 37.6 & 22.8 & 18.7 \\
\hline Western & 36.3 & 49.0 & 29.5 & 15.7 \\
\hline North Eastern & - & - & 4.1 & 0.5 \\
\hline Total & 34.2 & 37.2 & 24.9 & 25.5 \\
\hline
\end{tabular}

Source: Kenya DHS, 1993, 1998, 2003, 2008-09

Table 1b: Unwanted pregnancy by selected background characteristics (\%)

\begin{tabular}{|l|c|c|c|c|}
\hline $\begin{array}{l}\text { Background } \\
\text { Characteristics }\end{array}$ & $\mathbf{1 9 9 3}$ & $\mathbf{1 9 9 8}$ & $\mathbf{2 0 0 3}$ & $\mathbf{2 0 0 8 - 0 9}$ \\
\hline Age & & & & \\
\hline $15-24$ & 6.5 & 3.8 & 15.9 & 12.3 \\
\hline $25-34$ & 22.8 & 13.1 & 20.6 & 18.1 \\
\hline$>35$ & 39.1 & 40.7 & 47.6 & 44.6 \\
\hline Education & & & & 10.2 \\
\hline No education & 21.6 & 22.2 & 14.8 & 19.7 \\
\hline Primary & 17.0 & 11.0 & 20.9 & 13.8 \\
\hline Secondary + & 11.6 & 6.4 & 19.0 & \\
\hline
\end{tabular}




\begin{tabular}{|l|c|c|c|c|}
\hline Wealth & & & & \\
\hline Low & 15.7 & 12.4 & 18.9 & 18.5 \\
\hline Medium & 17.1 & 11.8 & 22.3 & 19.0 \\
\hline High & 17.5 & 8.8 & 19.5 & 14.9 \\
\hline Residence & & & & 11.4 \\
\hline Urban & 12.2 & 7.1 & 17.3 & 18.4 \\
\hline Rural & 17.4 & 11.9 & 20.1 & \\
\hline Region & & & & 14.5 \\
\hline Nairobi & 8.6 & 7.1 & 10.7 & 19.4 \\
\hline Central & 23.7 & 8.7 & 27.3 & 6.9 \\
\hline Coast & 4.9 & 6.1 & 10.5 & 22.6 \\
\hline Eastern & 22.6 & 17.3 & 23.1 & 18.2 \\
\hline Nyanza & 16.6 & 11.3 & 22.6 & 18.7 \\
\hline Rift Valley & 15.6 & 10.8 & 18.4 & 15.7 \\
\hline Western & 15.4 & 9.8 & 22.4 & 0.5 \\
\hline North Eastern & - & - & 0.4 & 17.1 \\
\hline Total & 16.7 & 11.1 & 19.6 & \\
\hline
\end{tabular}

Source: Kenya DHS, 1993, 1998, 2003, 2008-09

Table 1c: Unintended pregnancy (unwanted and mistimed) by selected background characteristics (\%)

\begin{tabular}{|c|c|c|c|c|}
\hline $\begin{array}{l}\text { Background } \\
\text { Characteristics }\end{array}$ & 1993 & 1998 & 2003 & 2008-09 \\
\hline \multicolumn{5}{|l|}{ Age } \\
\hline $15-24$ & 47.7 & 46.4 & 42.8 & 43.2 \\
\hline $25-34$ & 53.9 & 47.0 & 45.0 & 40.2 \\
\hline$>35$ & 53.3 & 62.8 & 58.9 & 57.0 \\
\hline \multicolumn{5}{|l|}{ Education } \\
\hline No education & 45.8 & 49.2 & 27.7 & 27.6 \\
\hline Primary & 53.8 & 51.0 & 49.1 & 47.7 \\
\hline Secondary + & 47.5 & 40.6 & 42.6 & 37.3 \\
\hline \multicolumn{5}{|l|}{ Wealth } \\
\hline Low & 48.4 & 50.8 & 44.5 & 45.2 \\
\hline Medium & 55.1 & 48.4 & 47.3 & 47.3 \\
\hline High & 51.5 & 44.7 & 43.7 & 37.9 \\
\hline \multicolumn{5}{|l|}{ Residence } \\
\hline Urban & 43.5 & 42.6 & 42.0 & 31.8 \\
\hline Rural & 52.0 & 49.5 & 45.1 & 45.1 \\
\hline \multicolumn{5}{|l|}{ Region } \\
\hline Nairobi & 44.6 & 36.4 & 38.5 & 29.0 \\
\hline Central & 54.6 & 38.2 & 45.3 & 38.8 \\
\hline Coast & 35.2 & 45.7 & 33.4 & 13.8 \\
\hline Eastern & 64.4 & 54.2 & 48.2 & 45.2 \\
\hline Nyanza & 43.9 & 45.6 & 55.4 & 36.4 \\
\hline Rift Valley & 49.6 & 48.4 & 41.2 & 37.4 \\
\hline Western & 51.7 & 58.8 & 51.9 & 31.4 \\
\hline North Eastern & - & - & 4.5 & 1.0 \\
\hline Total & 50.9 & 48.3 & 44.5 & 42.6 \\
\hline
\end{tabular}

Source: Kenya DHS, 1993, 1998, 2003, 2008-09 
Table 2: Percentage of currently married women 15-49 using contraceptive methods

\begin{tabular}{|l|c|c|c|c|}
\hline Method Used & $\mathbf{1 9 9 3}$ & $\mathbf{1 9 9 8}$ & $\mathbf{2 0 0 3}$ & $\mathbf{2 0 0 8 - 0 9}$ \\
\hline Any method & 32.7 & 39.0 & 39.3 & 45.5 \\
\hline Modern method & 27.3 & 31.5 & 31.5 & 39.4 \\
\hline Traditional method & 5.5 & 7.5 & 8.0 & 6.0 \\
\hline
\end{tabular}

Source: Kenya DHS, 1993, 1998, 2003, 2008-09

Table 2a: Percent distribution of all women age 15-49 using a method of contraception by background characteristics (Current users at the time of the survey)

\begin{tabular}{|c|c|c|c|c|c|c|c|c|}
\hline \multirow[b]{2}{*}{$\begin{array}{l}\text { Background } \\
\text { Characteristics }\end{array}$} & \multicolumn{4}{|c|}{ Any Modern Method } & \multicolumn{4}{|c|}{ Any Method } \\
\hline & 1993 & 1998 & 2003 & 2008-09 & 1993 & 1998 & 2003 & 2008-09 \\
\hline \multicolumn{9}{|l|}{ Age } \\
\hline $15-24$ & 14.1 & 16.4 & 13.8 & 16.5 & 9.1 & 11.4 & 10.6 & 14.1 \\
\hline $25-34$ & 37.9 & 40.8 & 38.7 & 44.7 & 31.8 & 32.7 & 31.5 & 40.5 \\
\hline$>35$ & 32.6 & 39.3 & 40.5 & 40.8 & 28.1 & 33.1 & 32.6 & 34.4 \\
\hline \multicolumn{9}{|l|}{ Education } \\
\hline No Education & 18 & 21.4 & 10.6 & 12.2 & 14.4 & 15.5 & 7.4 & 10.3 \\
\hline Primary Education & 23.6 & 26.3 & 27.1 & 31.7 & 19.0 & 21.0 & 21.5 & 27.8 \\
\hline Secondary + & 37.1 & 40.5 & 38.5 & 37.7 & 29.3 & 32.1 & 31.7 & 32.8 \\
\hline \multicolumn{9}{|l|}{ Wealth } \\
\hline Low & 14.3 & 19.5 & 18.4 & 21.8 & 10.6 & 13.4 & 13.1 & 18.3 \\
\hline Medium & 27.8 & 31.1 & 31.3 & 35.8 & 22.5 & 24.7 & 24.8 & 31.4 \\
\hline High & 35.8 & 39.2 & 35.3 & 38.6 & 29.2 & 32.8 & 30.3 & 34.2 \\
\hline \multicolumn{9}{|l|}{ Residence } \\
\hline Urban & 12.1 & 12.9 & 8.4 & 9.2 & 6.9 & 8.1 & 5.8 & 7.9 \\
\hline Rural & 32.7 & 39 & 39.3 & 45.5 & 27.3 & 31.5 & 31.5 & 39.4 \\
\hline Marital Status & 25.3 & 24.3 & 22.2 & 25.1 & 21.9 & 21.6 & 20.2 & 23.8 \\
\hline \multicolumn{9}{|l|}{ Never Married } \\
\hline Currently Married & 33.4 & 38 & 33.5 & 36.6 & 28.1 & 30.9 & 28.1 & 32.7 \\
\hline Formerly Married & 24.2 & 27.4 & 26.6 & 30.5 & 19.1 & 21.4 & 20.9 & 26.3 \\
\hline \multicolumn{9}{|l|}{ Province } \\
\hline Nairobi & 38.7 & 41.5 & 33.5 & 37.3 & 30.8 & 34.1 & 28.6 & 33.7 \\
\hline Central & 40.4 & 46.4 & 42.6 & 46.2 & 34.4 & 40.1 & 36.7 & 43.4 \\
\hline Coast & 17.1 & 18.0 & 20.2 & 26.7 & 14.3 & 16.3 & 16.0 & 23.6 \\
\hline Eastern & 29.7 & 34.6 & 35.5 & 36.1 & 22.1 & 26.2 & 27.3 & 30.4 \\
\hline Nyanza & 18.8 & 21.5 & 19.1 & 30.2 & 15.5 & 18.1 & 16.3 & 26.3 \\
\hline Rift Valley & 21.4 & 28.9 & 25.5 & 28.2 & 15.8 & 20.6 & 18.4 & 23.5 \\
\hline Western & 20.2 & 22.8 & 24.4 & 29.9 & 17.1 & 16.1 & 19.0 & 26.4 \\
\hline North Eastern & - & - & 0.1 & 2.4 & - & - & 0.1 & 2.4 \\
\hline Total & 27.3 & 31.5 & 31.5 & 39.4 & 32.7 & 39.0 & 39.3 & 45.5 \\
\hline
\end{tabular}

Source: Kenya DHS, 1993, 1998, 2003, 2008-09 
Table 3: Percentage of unmet need ${ }^{1}$ for family planning

\begin{tabular}{|l|c|c|c|c|}
\hline & $\mathbf{1 9 9 3}$ & $\mathbf{1 9 9 8}$ & $\mathbf{2 0 0 3}$ & $\mathbf{2 0 0 8 - 0 9}$ \\
\hline Currently Married Women & & & & \\
\hline Spacing & 20.7 & 16.0 & 15.2 & 12.5 \\
\hline Limiting & 14.6 & 11.9 & 12.2 & 13.1 \\
\hline Currently Unmarried Women & & & & \\
\hline Spacing & 3.5 & 7.6 & 3.8 & 4.2 \\
\hline Limiting & 2.3 & 2.2 & 3.8 & 3.0 \\
\hline All Women & & & & \\
\hline Spacing & 14.1 & 12.8 & 10.6 & 9.1 \\
\hline Limiting & 9.8 & 8.2 & 8.9 & 8.9 \\
\hline
\end{tabular}

Source: Kenya DHS, 1993, 1998, 2003, 2008-09

Table 3a: Trends in unmet need for FP among currently married women age 15-49 by background characteristics $(\%)$

\begin{tabular}{|c|c|c|c|c|c|c|c|c|}
\hline & \multicolumn{2}{|c|}{1993} & \multicolumn{2}{|c|}{1998} & \multicolumn{2}{|c|}{2003} & \multicolumn{2}{|c|}{ 2008-09 } \\
\hline & Spacing & Limiting & Spacing & Limiting & Spacing & Limiting & Spacing & Limiting \\
\hline \multicolumn{9}{|l|}{ Age } \\
\hline $15-24$ & 34.3 & 5.1 & 27.2 & 4.5 & 28.3 & 5.6 & 23.8 & 6.5 \\
\hline $25-34$ & 23.4 & 14.4 & 18.8 & 11.0 & 15.8 & 11.7 & 13.7 & 11.1 \\
\hline$>35$ & 6.6 & 22.3 & 5.0 & 18.4 & 4.4 & 17.9 & 3.4 & 20.1 \\
\hline \multicolumn{9}{|l|}{ Education } \\
\hline No Education & 16.5 & 18.7 & 10.7 & 18.2 & 11.3 & 12.6 & 16.5 & 10.0 \\
\hline Primary & 23.9 & 14.8 & 19.3 & 12.8 & 18.9 & 14.4 & 14.1 & 16.0 \\
\hline Secondary + & 16.8 & 9.5 & 11.9 & 6.9 & 9.0 & 7.3 & 7.9 & 8.6 \\
\hline \multicolumn{9}{|l|}{ Wealth } \\
\hline Low & 25.6 & 17.0 & 23.1 & 15.2 & 18.6 & 15.7 & 17.6 & 18.0 \\
\hline Medium & 21.2 & 16.0 & 13.3 & 11.4 & 13.5 & 12.9 & 10.2 & 11.6 \\
\hline High & 14.0 & 10.1 & 10.2 & 8.4 & 12.7 & 7.2 & 9.4 & 9.3 \\
\hline \multicolumn{9}{|l|}{ Residence } \\
\hline Urban & 13.7 & 10.1 & 11.8 & 8.6 & 11.6 & 7.9 & 10.7 & 8.9 \\
\hline Rural & 22.0 & 15.4 & 17.2 & 12.8 & 16.2 & 13.5 & 13.1 & 14.4 \\
\hline \multicolumn{9}{|l|}{ Province } \\
\hline Nairobi & 15.3 & 10.2 & 10.4 & 5.9 & 13.5 & 5.0 & 6.5 & 8.7 \\
\hline Central & 11.2 & 13.4 & 7.1 & 8.0 & 7.3 & 7.5 & 6.2 & 9.3 \\
\hline Coast & 24.8 & 8.4 & 21.4 & 12.5 & 16.9 & 10.7 & 15.9 & 9.3 \\
\hline Eastern & 21.8 & 18.3 & 14.0 & 13.5 & 12.6 & 12.4 & 9.8 & 14.0 \\
\hline Nyanza & 21.9 & 14.6 & 16.7 & 12.4 & 20.8 & 16.7 & 18.2 & 14.0 \\
\hline Rift Valley & 20.9 & 15.0 & 18.8 & 12.8 & 15.8 & 14.0 & 13.0 & 17.6 \\
\hline Western & 25.7 & 16.0 & 21.3 & 14.7 & 20.0 & 16.6 & 13.5 & 12.6 \\
\hline North Eastern & - & - & - & - & 9.1 & 1.3 & 17.1 & 0.8 \\
\hline Total & 20.7 & 14.6 & 16.0 & 11.9 & 15.2 & 12.2 & 12.5 & 13.1 \\
\hline
\end{tabular}

Source: Kenya DHS, 1993, 1998, 2003, 2008-09

\footnotetext{
${ }^{1}$ A woman has an unmet need if she is sexually active, does not want another child or wants to delay pregnancy, but she is not using any FP method.
} 
Table 3b: Percentage of contraceptive users who discontinued use of a method within 12 months of beginning its use

\begin{tabular}{|l|c|c|c|}
\hline Method & $\mathbf{1 9 9 8}$ & $\mathbf{2 0 0 3}$ & $\mathbf{2 0 0 8 - 0 9}$ \\
\hline Pill & 34.0 & 30.9 & 38.0 \\
\hline IUD & 9.0 & 11.7 & 6.4 \\
\hline Injectables & 18.1 & 22.9 & 28.0 \\
\hline Implants & - & 17.1 & 10.5 \\
\hline Male Condom & 61.5 & 35.1 & 71.6 \\
\hline Rhythm Method & 27.0 & 29.3 & 29.9 \\
\hline All Methods & $\mathbf{2 6 . 1}$ & $\mathbf{2 5 . 8}$ & $\mathbf{3 2 . 0}$ \\
\hline
\end{tabular}

Source: Kenya DHS, 1998, 2003, 2008-09

Table 3c: Method switching from first method choice, method choice by current contraceptive status and method use among all ever users (\%)

\begin{tabular}{|l|c|c|c|c|c|c|c|c|c|c|}
\hline $\begin{array}{l}\text { First } \\
\text { Method } \\
\text { Used }\end{array}$ & $\begin{array}{c}\text { Not Using } \\
\text { (Not Pregnant) }\end{array}$ & $\begin{array}{c}\text { Not Using } \\
\text { (Pregnant) }\end{array}$ & Pill & IUD & Inject. & Condoms & Steriliz. & Rhythm & Other & $\begin{array}{l}\text { No. of } \\
\text { Cases }\end{array}$ \\
\hline Pill & 35.0 & 7.2 & 23.6 & 3.2 & 18.3 & 1.4 & 6.7 & 3.0 & 1.5 & 1589 \\
\hline IUD & 24.9 & 3.4 & 8.9 & 31.6 & 12.2 & 2.1 & 10.5 & 4.6 & 1.7 & 237 \\
\hline Injections & 35.5 & 5.4 & 7.1 & 1.3 & 42.7 & 1.4 & 3.7 & 2.1 & 0.8 & 709 \\
\hline Condoms & 43.3 & 11.2 & 5.8 & 1.4 & 6.5 & 23.1 & 2.5 & 3.2 & 2.9 & 277 \\
\hline Sterilization & 0.0 & 0.0 & 0.0 & 0.0 & 0.0 & 0.0 & 100.0 & 0.0 & 0.0 & 147 \\
\hline Rhythm & 37.1 & 11.5 & 4.6 & 0.9 & 5.1 & 1.9 & 2.3 & 35.3 & 1.4 & 917 \\
\hline Other & 32.5 & 5.7 & 3.2 & 0.0 & 3.2 & 0.0 & 0.0 & 3.8 & 51.6 & 157 \\
\hline Total & $\mathbf{1 3 7 8}$ & $\mathbf{3 0 5}$ & $\mathbf{5 0 9}$ & $\mathbf{1 4 7}$ & $\mathbf{6 9 3}$ & $\mathbf{1 1 9}$ & $\mathbf{3 3 3}$ & $\mathbf{4 1 3}$ & $\mathbf{1 3 6}$ & $\mathbf{4 0 3 3}$ \\
\hline
\end{tabular}

Source: Kenya DHS, 1998

Table 3d: Women status 12 months after discontinuing for method-related reasons (\%)

\begin{tabular}{|r|c|c|c|c|c|c|c|}
\hline & & & & \multicolumn{2}{|c|}{ Current pregnancy or live birth } & \\
\hline & $\begin{array}{c}\text { No. of } \\
\text { discontinuations }\end{array}$ & $\begin{array}{c}\text { At } \\
\text { pregnancy } \\
\text { risk }\end{array}$ & Switched & Unwanted & Mistimed & Wanted & $\begin{array}{c}\text { Non-live } \\
\text { births }\end{array}$ \\
\hline All Methods & & & & & & & \\
\hline $\mathbf{1 9 9 8}$ & 604 & 28.9 & 36.8 & 7.0 & 15.6 & 9.2 & 2.4 \\
\hline $\mathbf{2 0 0 3}$ & 821 & 30.0 & 38.9 & 6.4 & 13.9 & 8.8 & 2.0 \\
\hline Modern Methods & & & & & & & \\
\hline $\mathbf{1 9 9 8}$ & 604 & 28.9 & 36.8 & 7.0 & 15.6 & 9.2 & 2.4 \\
\hline $\mathbf{2 0 0 3}$ & 821 & 30.0 & 38.9 & 6.4 & 13.9 & 8.8 & 2.0 \\
\hline
\end{tabular}

Ali MM, Cleland J, Shah IH. Causes and Consequences of Contraceptive Discontinuation: Evidence from 60 Demographic and Health Surveys. Geneva: World Health Organization; 2012. 
Table 3e: Women status 12 months after discontinuing for desire for pregnancy (\%)

\begin{tabular}{|c|c|c|c|c|c|c|c|}
\hline & & & & \multicolumn{3}{|c|}{ Current pregnancy or live birth } & \multirow[b]{2}{*}{$\begin{array}{l}\text { Non-live } \\
\text { birth }\end{array}$} \\
\hline & $\begin{array}{c}\text { No. of } \\
\text { discontinuations }\end{array}$ & At risk & Switched & Unwanted & Mistimed & Wanted & \\
\hline \multicolumn{8}{|l|}{ All Methods } \\
\hline 1998 & 358 & 12.6 & 0.0 & 4.1 & 11.5 & 67.1 & 4.7 \\
\hline 2003 & 391 & 18.6 & 0.8 & 2.2 & 3.6 & 72.0 & 2.8 \\
\hline \multicolumn{8}{|l|}{ Modern Methods } \\
\hline 1998 & 243 & 16.0 & 0.0 & 3.4 & 13.9 & 61.4 & 5.2 \\
\hline 2003 & 257 & 23.0 & 1.2 & 1.7 & 2.1 & 69.1 & 2.8 \\
\hline \multicolumn{8}{|l|}{ Traditional Methods } \\
\hline 1998 & 114 & 5.5 & 0.0 & 5.5 & 6.4 & 79.0 & 3.6 \\
\hline 2003 & 133 & 10.1 & 0.0 & 3.0 & 6.5 & 77.6 & 2.7 \\
\hline
\end{tabular}

Ali MM, Cleland J, Shah IH. Causes and Consequences of Contraceptive Discontinuation: Evidence from 60 Demographic and Health Surveys. Geneva: World Health Organization; 2012.

Figure 1: Trends in annual contraceptive failure rates

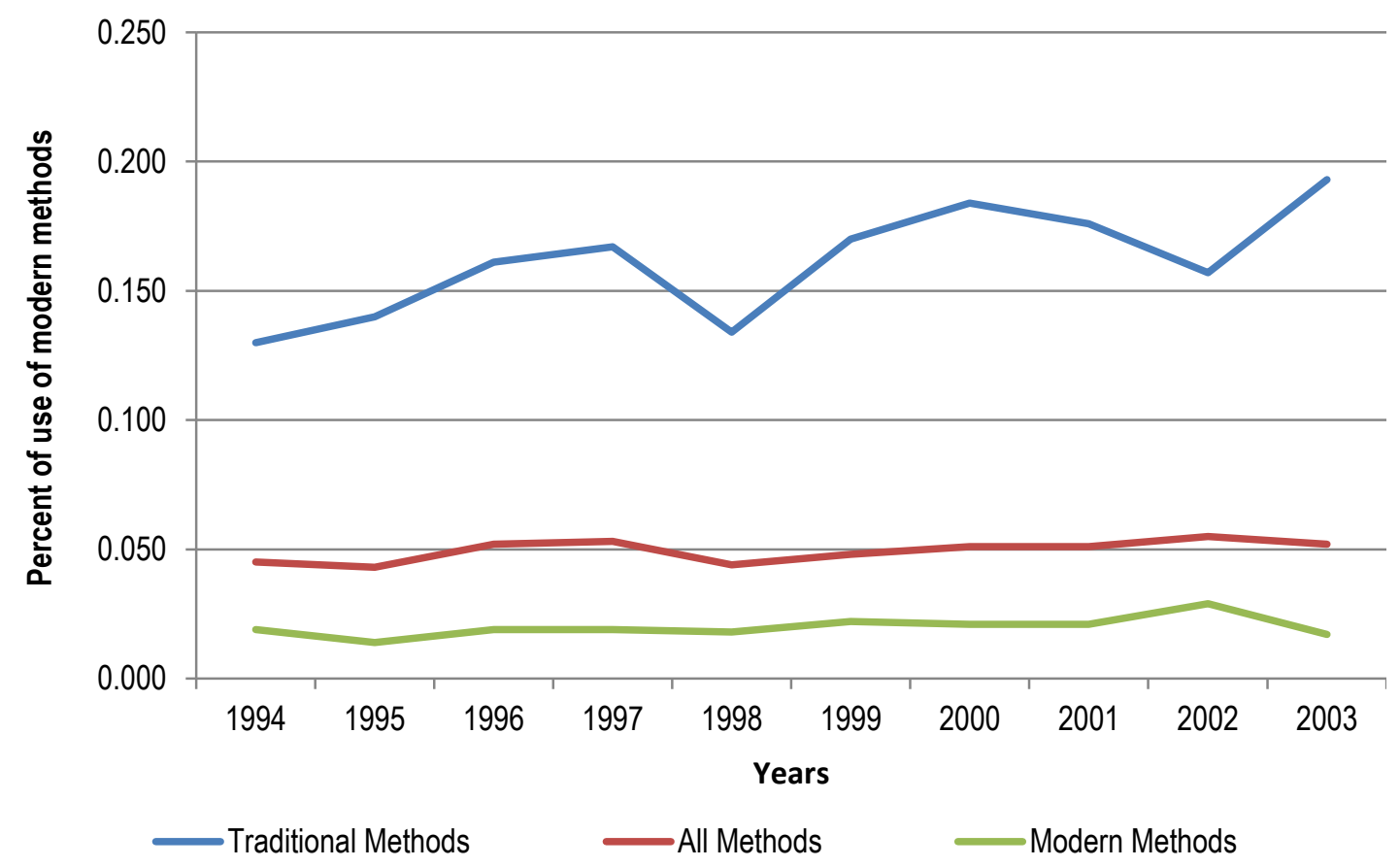

Source: Westoff, Charles F., and Anne R. Cross. 2006. The Stall in the Fertility Transition in Kenya. DHS Analytical Studies No. 9. Calverton, Maryland: ORC Macro.

Table 4: Contraceptive users that discontinued after the first year of use for reasons other than desire for pregnancy $(\%)$

\begin{tabular}{|l|c|c|}
\hline Method & $\mathbf{1 9 9 8}$ & $\mathbf{2 0 0 3}$ \\
\hline Pill & 32 & 42 \\
\hline Injectables & 18 & 28 \\
\hline Condom & 54 & 53 \\
\hline Periodic abstinence & 27 & 27 \\
\hline All methods & $\mathbf{2 8}$ & $\mathbf{3 3}$ \\
\hline
\end{tabular}

Source: Kenya DHS, 1998 and 2003 
Table 4a: Reproductive consequences of reported contraceptive failure (\%)

\begin{tabular}{|c|c|c|c|c|c|}
\hline & \multirow[b]{2}{*}{ No. of failures } & \multicolumn{3}{|c|}{ Current pregnancy or live birth } & \multirow[b]{2}{*}{ Non-live birth } \\
\hline & & Unwanted & Mistimed & Wanted & \\
\hline \multicolumn{6}{|l|}{ All Methods } \\
\hline 1998 & 243 & 20.3 & 52.0 & 23.0 & 4.7 \\
\hline 2003 & 272 & 27.7 & 49.8 & 16.9 & 5.6 \\
\hline \multicolumn{6}{|l|}{ Modern Methods } \\
\hline 1998 & 68 & 26.7 & 49.7 & 19.9 & 3.6 \\
\hline 2003 & 94 & 31.0 & 55.1 & 10.7 & 3.2 \\
\hline \multicolumn{6}{|l|}{ Traditional Methods } \\
\hline 1998 & 174 & 17.8 & 52.8 & 24.2 & 5.2 \\
\hline 2003 & 178 & 26.0 & 46.9 & 20.2 & 6.9 \\
\hline
\end{tabular}

Source: Kenya DHS, 1998 and 2003

Table 5: Trends in Wanted Fertility Rates (WFR), Unwanted Fertility Rates (UWFR) and Total Fertility Rate (TFR) for the three years preceding the survey

\begin{tabular}{|c|c|c|c|c|}
\hline & $\mathbf{1 9 9 3}$ & $\mathbf{1 9 9 8}$ & $\mathbf{2 0 0 3}$ & $\mathbf{2 0 0 8 - 0 9}$ \\
\hline Unwanted Fertility Rates & 2.4 & 1.5 & 1.3 & 1.4 \\
\hline Wanted Fertility Rates & 3.0 & 3.2 & 3.6 & 3.2 \\
\hline Total Fertility Rate & $\mathbf{5 . 4}$ & $\mathbf{4 . 7}$ & $\mathbf{4 . 9}$ & $\mathbf{4 . 6}$ \\
\hline
\end{tabular}

Source: Kenya DHS, 1993, 1998, 2003, 2008-09

Table 6. Estimated number of cases of induced abortion complications and overall induced abortions by region, Kenya, 2012

\begin{tabular}{|l|c|c|c|c|}
\hline \multicolumn{2}{|c|}{} & \multicolumn{2}{c|}{ Number of all induced abortions } \\
\hline Region & $\begin{array}{c}\text { No. of women treated } \\
\text { for induced abortion } \\
\text { complications at } \\
\text { health facilities }\end{array}$ & Low estimate & Medium estimate & High estimate \\
\hline Central \& Nairobi & 20,676 & 49,828 & 70,504 & 91,180 \\
\hline Coast and North Eastern & 16,649 & 49,616 & 66,265 & 82,914 \\
\hline Eastern & 7,057 & 21,030 & 28,087 & 35,144 \\
\hline Nyanza \& Western & 36,842 & 109,789 & 146,631 & 183,473 \\
\hline Rift Valley & 38,687 & 115,289 & 153,976 & 192,664 \\
\hline Total & $\mathbf{1 1 9 , 9 1 2}$ & $\mathbf{3 4 4 , 7 7 8}$ & 464,690 & $\mathbf{5 8 4 , 6 0 1}$ \\
\hline
\end{tabular}

Source: African Population and Health Research Center, Ministry of Health (MOH), Kenya, Ipas, and Guttmacher Institute. Incidence and Complications of Unsafe Abortion in Kenya: Key Findings of a National Study. Nairobi, Kenya. APHRC, Ministry of Health (MOH), Kenya, Ipas, and Guttmacher Institute. 2013. 
Table 6a. National and regional induced abortion rates and ratio, Kenya, 2012

\begin{tabular}{|l|c|c|c|}
\hline Region & $\begin{array}{c}\text { Number of Women } \\
\text { (in 1,000's) of } \\
\text { reproductive age (15-49) }\end{array}$ & $\begin{array}{c}\text { Induced Abortion Rate } \\
\text { per 1,000 women of } \\
\text { reproductive age }\end{array}$ & $\begin{array}{c}\text { Induced Abortion } \\
\text { Ratio per 100 live } \\
\text { births }\end{array}$ \\
\hline Central \& Nairobi & 2186 & 32 & 20 \\
\hline Coast and North Eastern & 1298 & 51 & 32 \\
\hline Eastern & 1382 & 20 & 13 \\
\hline Nyanza \& Western & 2329 & 63 & 39 \\
\hline Rift Valley & 2404 & 64 & 40 \\
\hline Total & $\mathbf{9 6 0 0}$ & $\mathbf{4 8}$ & $\mathbf{3 0}$ \\
\hline
\end{tabular}

Source: African Population and Health Research Center, Ministry of Health (MOH), Kenya, Ipas, and Guttmacher Institute. Incidence and Complications of Unsafe Abortion in Kenya: Key Findings of a National Study. Nairobi, Kenya. APHRC, Ministry of Health (MOH), Kenya, Ipas, and Guttmacher Institute. 2013.

Table 6 b. Socio-demographic characteristics of women seeking PAC in health facilities over a 30-day period, Kenya, 2012

\begin{tabular}{|l|c|c|}
\hline Characteristics & $\%$ & N \\
\hline Age & & \\
\hline $10-19$ Years & 16.5 & 335 \\
\hline $20-24$ Years & 31.5 & 810 \\
\hline $25+$ Years & 51.3 & 1478 \\
\hline Residence & & \\
\hline Urban & 40.9 & 1386 \\
\hline Rural & 58.7 & 1238 \\
\hline Marital Status & & \\
\hline Never Married & 27.8 & 761 \\
\hline Married/Living together & 64.4 & 1700 \\
\hline Divorced & 7.5 & 162 \\
\hline Education & & \\
\hline No education & 9.3 & 158 \\
\hline Primary & 40.1 & 963 \\
\hline Secondary & 35.5 & 952 \\
\hline Post-secondary & 14.8 & 543 \\
\hline Occupation & & \\
\hline Farmer/unskilled & 24.9 & 589 \\
\hline Skilled/clerical & 20.2 & 684 \\
\hline Student & 13.0 & 384 \\
\hline Unemployed/housewife & 41.7 & 965 \\
\hline Religion & & \\
\hline Catholic & 24.3 & 606 \\
\hline Other Christians & 64.4 & 1767 \\
\hline Muslims & 8.5 & 216 \\
\hline Others & 2.3 & 30 \\
\hline Total & 100 & 2631 \\
\hline Souce: Afian Pop & & \\
\hline
\end{tabular}

Source: African Population and Health Research Center, Ministry of Health (MOH), Kenya, Ipas, and Guttmacher Institute. Incidence and Complications of Unsafe Abortion in Kenya: Key Findings of a National Study. Nairobi, Kenya. APHRC, Ministry of Health (MOH), Kenya, Ipas, and Guttmacher Institute. 2013. 


\section{References}

1. African Population and Health Research Center (APHRC), Ministry of Health (MOH), Ipas, Guttmacher Institute (2013). Incidence and complications of unsafe abortions in Kenya: Key findings of a national study. Nairobi, Kenya: APHRC, $\mathrm{MOH}$, Ipas, Guttmacher Institute.

2. Ali MM, Cleland J, Shah $\mathrm{H}$. Causes and Consequences of Contraceptive Discontinuation: Evidence from 60 Demographic and Health Surveys. Geneva: World Health Organization; 2012.

3. APHRC (2001). Contraceptive Use Dynamics in Kenya Further Analysis of Demographic and Health Survey (DHS) Data, Nairobi, Kenya, Macro International Inc, Calverton, Maryland USA

4. Central Bureau of Statistics (CBS) [Kenya], Ministry of Health (MOH) [Kenya], and ORC Macro, Kenya Demographic and Health Survey 2003 (Calverton, MD: CBS, MOH, and ORC Macro, 2004).

5. Central Bureau of Statistics. (1993). Kenya Demographic Health Survey, Nairobi: Ministry of Planning and National Development, Kenya.

6. Central Bureau of Statistics. (1998). Kenya Demographic Health Survey. Nairobi: Ministry of Planning and National Development, Kenya.

7. KNBS \& ICF Macro. (2010). Kenya Demographic and Health Survey 2008-09. Calverton, Maryland: KNBS and ICF Macro.

8. Mumah, J, Kabiru, CW, Mukiira, C, Brinton, J, Mutua, M, Izugbara, C, Birungi, H. and Askew, I. (2014). "Unintended Pregnancies in Kenya: A Country Profile," STEP UP Research Report. Nairobi: African Population and Health Research Center.

9. Westoff, Charles F., and Anne R. Cross. (2006). The Stall in the Fertility Transition in Kenya. DHS Analytical Studies No. 9. Calverton, Maryland: ORC Macro.

The STEP UP (Strengthening Evidence for Programming on Unintended Pregnancy) Research Programme Consortium generates policy-relevant research to promote an evidence-based approach for improving access to family planning and safe abortion. STEP UP focuses its activities in five countries: Bangladesh, Ghana, India, Kenya, and Senegal. STEP UP is coordinated by the Population Council in partnership with the African Population and Health Research Center; ICDDR,B; the London School of Hygiene and Tropical Medicine; Marie Stopes International; and Partners in Population on Development. STEP UP is funded by UKaid from the Department for International Development.

For more information on STEP UP, please contact:

Dr. Harriet Birungi, hbirungi@popcouncil.org

Dr. lan Askew, iaskew@popcouncil.org

(C) 2014 African Population and Health Research Center

Funded by

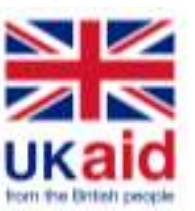

